\title{
Lobectomy or pneumonectomy for multidrug-resistant pulmonary tuberculosis can be performed with acceptable morbidity and mortality: A seven-year review of a single institution's experience
}

\author{
Tarek Mohsen, MD, FRCS, ${ }^{a}$ Amany Abou Zeid, MD, MRCP, and Saleem Haj-Yahia, MD, BSc ${ }^{\mathrm{c}}$
}

From the Departments of Cardiothoracic Surgery $^{\mathrm{a}}$ and Chest Medicine, ${ }^{\mathrm{b}}$ Kasr El Aini Hospital, Cairo University, Cairo, Egypt; and the Department of Cardiothoracic Surgery, ${ }^{\mathrm{c}}$ Royal Brompton and Harefield Hospital, National Heart and Lung Institute, Imperial College London, United Kingdom.

Received for publication Nov 15, 2006; revisions received Feb 13, 2007; accepted for publication March 29, 2007.

Address for reprints: Saleem Haj-Yahia, MD, BSc, Cardiothoracic Transplantation Surgery and Mechanical Circulatory Support, Royal Brompton \& Harefield Hospital, National Heart \& Lung Institute, Imperial College London, London, United Kingdom (Email address: s.haj-yahia@ imperial.ac.uk).

J Thorac Cardiovasc Surg 2007;134:194-8

$0022-5223 / \$ 32.00$

Copyright (๑) 2007 by The American Association for Thoracic Surgery

doi:10.1016/j.jtcvs.2007.03.022
Objective: Combination chemotherapy is considered the first-line treatment for pulmonary tuberculosis. Despite related morbidity, the need for surgical resections coincides with the emergence of multidrug-resistant tuberculosis. This study presents a single-institution retrospective audit of the surgical management of 23 patients with multidrug-resistant tuberculosis.

Methods: We analyzed 23 consecutive patients undergoing anatomic pulmonary resections for human immunodeficiency virus-negative multidrug-resistant tuberculosis. Twenty were male (87\%) and 3 were female (13\%); their mean age was 24.4 years. We defined resistance in this cohort as failure to respond to combination chemotherapy, including isoniazid and rifampicin, with a mean duration of administration being 90 days. Fifteen of 23 (65.3\%) patients, although sputum negative, were considered at risk for relapse owing to extensive parenchymal disease. Eight (34.7\%) of 23 patients were sputum positive at the time of operation. We performed pneumonectomy on $11(47.8 \%)$ and lobectomy on 12 (52\%) patients. All had adjuvant chemotherapy for 18 to 24 months, with follow-ups ranging from 14 to 27 months.

Results: Stay in the intensive treatment unit was 2.9 days (range 1-17 days) and hospital stay, 8.6 days (range 5-45 days). Four (17\%) patients had prolonged air leak, $3(13 \%)$ required further treatment for empyema, with re-exploration for bleeding in 1 (4\%). Hospital mortality was $4.3 \%$. All patients attained sputumnegative status postoperatively (range 1-5 months). One (4\%) patient had a relapse after 12 months.

Conclusion: Surgery should be considered as an adjunct to medical therapy when eradicating multidrug-resistant tuberculosis in affected patients. Anatomic lung resections can be performed with acceptable morbidity and mortality. Early referral of such patients for surgical consideration is warranted.

$\mathrm{T}$ he incidence of tuberculosis in Egypt is 27 per 100,000 population per year. The success of the authorities in controlling infection by adopting a policy of directly observed treatment has reached $80 \%$. However, the prevalence of newly diagnosed cases of multidrug-resistant tuberculosis (MDR-TB) is approximately $2.2 \%$. If treatment is subtherapeutic, this estimate climbs to $7.1 \%$. ${ }^{1}$ Surgery for pulmonary tuberculosis was an important form of therapy until the introduction of antibiotics in the 1960s. The benefits of appropriate chemotherapy outweighed surgery, with a cure achieved in all patients with tuberculosis. ${ }^{2,3}$ The emergence of strains resistant to rifampicin and isoniazid, however, as well as of such atypical mycobacteria as Mycobacterium avium-intracellulare, has questioned the completeness of the benefits of medical therapy. Surgical options have once again emerged 


\section{Abbreviations and Acronyms \\ MDR-TB = multidrug-resistant tuberculosis}

as a necessary treatment modality. ${ }^{4,5}$ In this study, we retrospectively analyzed the indications, surgical outcomes, complications, and follow-up of patients with MDR-TB.

\section{Materials and Methods}

Between 1999 and 2005, 23 patients underwent pulmonary resections at Cairo University Hospital for human immunodeficiency virus-negative stage III MDR-TB. Twenty (87\%) were male and 3 $(13 \%)$ were female, with a mean age of 24.4 years (range $7-48$ years).

Preoperative evaluation included history and clinical examination to address any comorbidity, particularly underlying lung disease (ie, bronchiectasis), diabetes, anemia, and hypoalbuminemia, involving the performance of chest radiography, computed tomography, and bronchoscopy to rule out the rare endobronchial tuberculosis, and pulmonary function tests and echocardiograms to rule out pulmonary hypertension.

All patients in whom sputum conversion failed, following World Health Organization recommendations, started with 4 drugs for 2 months of a directly observed treatment short course. At 4 months of persistent sputum positivity we considered the treatment to have failed. At this stage we tested for culture and drug sensitivity with the BACTEC TB 460 assay (Becton Dickinson, Franklin Lakes, NJ). All patients were resistant to a median of 4 drugs (range 2 to 6 drugs), including rifampicin and isoniazid. In the preoperative period all patients were placed on a regimen of multidrug therapy, with a median of 4 drugs (range 3-6 drugs), based on appropriate chemotherapy sensitivity profiling. Pharmacologic agents included pyrazinamide, ethambutol, streptomycin, para-aminosalicylic acid, and fluoroquinolones (floxacin, levofloxacin, and gatifloxacin), the latter used in 15 (65.3\%) of 23 patients in this study. The regimen was given for at least 3 months (range 3-6 months).

The criteria for patient selection and indications for surgical resections as adjunct to medical treatment were (1) drug resistance to at least 2 drugs (isoniazid and rifampicin), (2) failure to convert sputum or a previous relapse in compliant patients, (3) sputumnegative patients with a high probability of relapse owing to considerable parenchymal destruction (cavitary lesion or destroyed lung), (4) the availability of adequate drug efficacy to provide rapid healing of the bronchial stump, (5) the disease being sufficiently localized so that resection would leave an adequate cardiopulmonary capacity, and (6) the operation being performed after neoadjuvant chemotherapy for at least 3 months (range 3-6 months). ${ }^{5}$

We performed 11 pneumonectomies (10 on the left and 1 on the right) and 12 upper lobectomies ( 8 on the right and 4 on the left). We have operated unilaterally on this series of patients, targeting such gross diseases as cavity and destroyed lobes, or managing such complications as hemoptysis or mycetoma. The operation was performed under general anesthesia, with double-lumen endotracheal tubes used to prevent contamination of the nonoperated
TABLE 1. Clinical profile of 23 patients with MDR-TB

\begin{tabular}{|c|c|}
\hline Age (mean), y & $24.4(7-48)$ \\
\hline Gender & $20 \mathrm{M} / 3 \mathrm{~F}$ \\
\hline Body mass index & $20.43 \pm 2.84$ \\
\hline \multicolumn{2}{|l|}{ Origin, $\mathrm{n}$} \\
\hline Egyptian & 9 \\
\hline Yemeni & 7 \\
\hline Sudani & 4 \\
\hline Libyan & 3 \\
\hline \multicolumn{2}{|l|}{ Preop associated complications, $\mathrm{n}$} \\
\hline Hemoptysis & 4 \\
\hline Fungus ball & 3 \\
\hline \multicolumn{2}{|l|}{ X-ray findings, $\mathrm{n}(\%)$} \\
\hline Cavity & $9(39.1 \%)$ \\
\hline Bronchiectasis & $3(13 \%)$ \\
\hline Destroyed lung & $11(37.8 \%)$ \\
\hline Bilateral lesions & $4(17.3 \%)$ \\
\hline Median No. of resistant drugs & $4(3-6)$ \\
\hline Preop duration of medical treatment, mo & $3-6$ \\
\hline Preop organisms identified & $\begin{array}{c}\text { Mycobacterium } \\
\text { tuberculosis }\end{array}$ \\
\hline Preop positive sputum, No. patients (\%) & $8 / 23(34.7 \%)$ \\
\hline \multicolumn{2}{|l|}{ Preop pulmonary reserve } \\
\hline FVC (L) & $3.15 \pm 0.75$ \\
\hline FVC $\%$ of predicted $(\%)$ & $100.6 \pm 18.9$ \\
\hline $\mathrm{FEV}_{1.0}$ & $2.23 \pm 0.82$ \\
\hline $\mathrm{FEV}_{1.0} \%$ of predicted $(\%)$ & $92.5 \pm 28.4$ \\
\hline Median postop conversion time, mo & $3(1-5)$ \\
\hline
\end{tabular}

MDR-TB, Multidrug-resistant tuberculosis; FVC, forced vital capacity; $F E V_{1.0}$, forced expiratory volume in 1 second.

lung through the bronchial tree. In all patients the approach was through a standard muscle-sparing, posterolateral thoracotomy. The dissection of dense adhesions in some patients was not possible and an extrapleural approach was used to avoid opening cavitary lesions and to reduce blood loss (in 7 of 11 pneumonectomies). The use of the Harmonic scalpel (Ethicon, Inc, Somerville, NJ) also contributed to the reduction of blood loss. All bronchial stumps were sutured and reinforced with pleural flaps or pericardial fat pads. Warm saline was used to irrigate the pleural cavity, together with povidone-iodine. All specimens were sent for pathologic and bacteriologic evaluation.

\section{Postoperative Care}

All patients were weaned from the ventilator immediately after the operation in the operating theater. We did not use suction and depended on proper analgesia using epidural catheters, physiotherapy, incentive spirometry, and early ambulation (6-8 hours postoperatively).

All patients recommenced their preoperative multidrug regimen as soon as they resumed oral intake. They were transferred from the high dependency unit to the thoracic surgical ward once hemodynamically stable and after the removal of all chest drains. We followed a protocol of early removal of chest drains after pneumonectomy as long as 
drainage was less than $2 \mathrm{~mL} / \mathrm{kg}$ in 24 hours. No alteration in individual multidrug regimens was necessary, following reviews of specimen cultures and drug susceptibility profiles. The follow-up period extended from 14 to 27 months. Drugs were administered for from 18 to 24 months, except in 1 case, in which drugs were administered for 12 months only.

\section{Results}

Patients' preoperative demographics and variables are shown in Table 1. There was 1 (4.3\%) perioperative death. This patient, who had undergone right pneumonectomy for a destroyed lung, had a high fever, and an empyema was diagnosed on the fifth postoperative day. His condition was complicated by esophageal perforation. On the 11th postoperative day a bronchopleural fistula developed and he needed mechanical ventilation. His condition continued to deteriorate and he died on the 17th postoperative day of multiorgan failure.

Postoperative complications occurred in $8(34.7 \%)$ patients. The major complication was prolonged air leakage for more than 7 days (range 11 days to 7 months) in 4 (33.3\%) of 12 patients having lobectomy. The air leaks developed mostly from the fissure area.

One patient had incomplete fissure and an air leak was inevitable; then the lung failed to expand, and we reexplored the hemithorax 10 days later. At the operation there was a peel hindering lung expansion and a small area of air leakage at the fissure. The peel was dissected and the site of the air leak was hand sewn. Lung expansion was achieved and the patient had an uneventful postoperative course and was discharged home a week later.

We treated the other 3 conservatively. Two responded to repeated autologous blood. One hundred fifty milliliters of autologous blood was injected through the anterior chest tube for 2 successive days, while clamping for 1 hour each time. Both patients' leaks became minimal after the first injection and stopped after the second. The last patient refused any further intervention and was discharged home with a Heimlich valve; her air leak stopped 7 months later.

Of the survivors, 3 patients had empyema. One patient had a high fever and elevated white blood cell count on day 5 after left pneumonectomy. The bacteriologic culture from the thin turbid fluid tapped revealed Staphylococcus aureus, which was sensitive to vancomycin. A wide-bore dependent thoracostomy tube was placed, and systemic as well as local vancomycin in $2 \mathrm{~L}$ of warm saline was given 3 times a day via a central venous catheter placed in the second intercostal space. This was done for 5 days; repeated cultures taken from the drains were negative. The tube was removed on day 15 and the patient was discharged home.

The second patient had empyema after a right upper lobectomy. Drains were kept in place, and no organism was detected on repeated cultures. Although full lung expansion was achieved, multiple fluid levels developed and localization occurred with a posterior pouch. When drainage was minimal (less than $2 \mathrm{~mL} / \mathrm{kg}$ in 24 hours) after 4 weeks, re-exploration, debridement, and decortication were performed and achieved good results. The patient was discharged 10 days later.

The third patient had a posterior pouch on day 9 after the drains were removed. However, pleural aspiration revealed that turbid fluid and Escherichia coli had been cultured. The pouch was cleared and the peel was dissected by videoassisted thoracoscopy aided by manual lung bagging. A dependent wide-bore drain was placed and a lavage with antibiotics was done for 5 days. The patient was discharged home on day 2.

One patient underwent re-exploration after 24 hours owing to high drainage, and at operation no source of bleeding was found and a large hematoma was evacuated.

Four patients with bilateral disease $(17.3 \%)$ were managed unilaterally with upper lobectomies. Three had fungal balls and 1 had upper lobe bronchiectasis, which was the source of hemoptysis (confirmed by bronchoscopy). In these patients the contralateral side had minimal infiltrations that responded to medical therapy at follow-up.

The organism identified preoperatively in all cases was Mycobacterium tuberculosis. The incidence of positive and negative sputum at the time of the operation was $65.3 \%$ $(\mathrm{n}=15)$ and $34.7 \%(\mathrm{n}=8)$, respectively. All survivors were culture negative after the operation, within a median of 3 months (range 1-5 months). One patient had a relapse after 12 months of retreatment. He discontinued the treatment and returned with symptoms. His culture was positive and we lost him to follow-up.

\section{Discussion}

The emergence of MDR-TB is a global problem. ${ }^{6}$ Its rising incidence in developed countries, and more recently even in developing countries, has been attributed to immigration, homelessness, intravenous drug abuse, and acquired immunodeficiency syndrome. On the other hand, the problem in developing countries is attributed to poor patient compliance, illiteracy, and inadequate follow-up, thus allowing drug resistance and the progression of the disease to occur. ${ }^{7}$ It has been estimated that the cost of treating drug-sensitive tuberculosis may range from $\$ 2,000$ to $\$ 15,000$ per patient. This pales in comparison with the treatment of MDR-TB, which may approach $\$ 200,000 .{ }^{4}$

To date there is no randomized, controlled study comparing the efficacy of surgical treatment plus chemotherapy with the efficacy of chemotherapy alone. However, the failure rate of medical therapy is calculated to be more than $40 \%$, with a poor long-term survival reported. ${ }^{8}$ The cure rate with surgical resectioning as an adjunct to medical 
therapy approximates $83 \%$ to $93 \%$ in some published series. ${ }^{8,9}$ Surgical treatment reduces the bacterial burden by resecting cavitary lesions or destroyed lobes. It may also manage such complications of persistent infection as hemoptysis, bronchopleural fistulas, empyema, or persistent localized infection in destroyed lungs or distal to a bronchostenosis. Our indications for surgery in patients with MDR-TB are similar to those of others, ${ }^{5,10-15}$ presuming that there is adequate cardiopulmonary reserve. It is important to note that at least 3 months of treatment should be given before surgery, if at all feasible. ${ }^{4,9,16}$ The introduction of fluoroquinolone antibiotics has raised hopes of improving the current dismal prognosis for MDR-TB. ${ }^{9}$

The indications for surgery were as follows:

1. Drug resistance to at least 2 drugs, including isonaizid and refampicin; this was discovered after drug susceptibility in patients who failed to convert from sputum-positive smears to sputum-negative smears

2. Previous relapse(s) in patients with histories of tuberculosis and proper drug regimen

3. Sputum-negative results in patients who have a high probability of relapse owing to such gross lung diseases as bronchiectasis, cavitary lung lesions, or destroyed lungs or lobes

4. The availability of a drug regimen to provide rapid healing of the bronchial stump. This indication is essential in patients with rare endobronchial tuberculosis. However, we did not encounter that in our series. Moreover, 8 of our patients were sputum positive at operation, but still we did not experience stump problems

5. Resectioning confined to localized disease, provided that adequate cardiopulmonary function was left

6. An umbrella of susceptible drug regimen for at least 3 months or more could precede surgical intervention

When the probability of the negative bacteriologic conversion of sputum seems high, such as with less-destroyed lungs, initial resistance to isoniazid and rifampicin, or resistance only to isoniazid and rifampin, chemotherapy should be continued for an additional 2 to 3 months. Should any significant changes occur clinically, chemotherapy should continue, whereas no change or a worsening in clinical, radiologic, or bacteriologic findings indicates surgery.

The mortality rate of $4.3 \%$ in our group was slightly higher than that of other reported groups. The Denver group reported $6(3.3 \%)$ of 180 patients, ${ }^{4}$ and such other groups as the Japanese (30 patients), ${ }^{9}$ Turks (27 patients), ${ }^{2}$ and Koreans (27 patients) ${ }^{16}$ reported no early mortality.

Fluoroquinolones, particularly moxifloxacin and gatifloxacin, are believed to have the most activity in vitro against Mycobacterium tuberculosis, followed by levofloxacin and ofloxacin. ${ }^{17-19} \mathrm{We}$ used fluoroquinolones, mostly levofloxacin and gatifloxacin, in $66.6 \%$ of our pa- tients $(15 / 23)$, and $66.6 \%$ of the patients could be converted to being sputum negative preoperatively. These results are similar to the results of Shiraishi and associates, ${ }^{9}$ who reported a high conversion rate with levofloxacin used in a multidrug regimen.

Major complications after surgery for MDR-TB were reported in between $15 \%$ and $40 \%$ of patients in the studies. $^{2-4,9}$ In our series, major complications occurred in $34.7 \%$ ( $8 / 23$ patients). Half of our complications were as a result of prolonged air leaks that, except in 1 patient, were managed conservatively. The high incidence of air leakage was a result of two factors.

First, it is a disease of the lung periphery, with extensive vascular dense adhesions that affect the whole hemithorax (stage TB) and are not confined to the diseased lobe only. In this group of patients, complete dissection of the lung was mandatory to ensure residual lung expansion. Subsequent dissections over unhealthy lower lobes were a source of air leak.

Second, the residual lobe or lobes after resection were also pathologic, and lung expansion was not feasible, such as in patients with bronchogenic carcinoma. These factors are considerable in patients with tuberculosis. Although an extrapleural approach was adopted for many of our patients, this was confined to the area of resected lobe. The main goal was to avoid bleeding and opening cavities, but not air leak. Among 4 patients who had air leak, 1 had an incomplete fissure and the rest had air leak at the fissure site, an area that is usually difficult to control by staples or stitches.

Although use of biological glue has been reported with good results in other centers, our experience of treating postoperative air leak by infusions of $150 \mathrm{~mL}$ of autologous blood, without the use of BioGlue surgical adhesive (CryoLife, Inc, Kennesaw, Ga) is extensive and yields an almost complete resolution. We used expectant treatment in 3 of 4 of our patients, and only the treatment for the patient with an incomplete fissure had to be revised. For this particular patient, we used stitches over two pericardial strips, reinforced with biological glue. One patient had air leakage for 7 months, but she refused any interventions.

Similar to the techniques of other surgeons, ${ }^{2}$ we did not use any muscle flaps to secure bronchial stumps because it was hard to expect the presence of a large muscle bulk owing to malnutrition problems. However, we were able to use pleural flaps or pericardial fat pad to cover all bronchial stumps after resection in patients treated by pneumonectomy and lobectomy. Except in our patient who died, we did not encounter any bronchopleural fistulas, serious space problems, or difficult empyemas that were not responding to the first line of management, which was composed of adequate drainage, antibiotics, and, in 1 patient, debridement. We attribute this to our policy of avoiding extensive bronchial dissection and manipulation. 
Staple technique reduces time when compared with hand-sewn technique. However, apart from that, previous reports have shown no major advantages of staple over hand-sewn technique. Our preferred sewing method was the hand-sewn technique, mainly because of lower cost.

For postoperative care we adopted an early extubation policy and did not use suction on the chest drains. Adequate analgesia, using epidural catheters, physiotherapy, incentive spirometry, and early ambulation, played an important role in achieving a higher success rate.

The majority of this series of patients has shown satisfaction on long-term follow-up. Those with hemoptysis or considerable sputum production (large cavities, destroyed lungs) were among those who enjoyed the most benefit. We did not encounter any patient who reported having significant dyspnea or exercise intolerance. To the contrary, most of them had gained weight and had a general feeling of well-being.

Our success in this series is $91 \%$, with 1 patient experiencing relapse and another dying after the operation. The success rate in other series has been reported to range between $83 \%$ and $93 \% .^{4,9}$

In summary, surgery remains an important adjunct tool in the management of patients with MDR-TB. The success of surgery depends on good cooperation between chest physicians and thoracic surgeons, patient compliance in completing the postoperative drug regimen of 18 to 24 months, surgical experience, adherence to preoperative and postoperative precautions, and careful follow-up.

\section{References}

1. World Health Organization TB country profile. Available from: http://www.who.int/GlobalAtlas/predefined\%20Reports/TB/PDF_Files/ EG_2004_Brief.pdf. http://www.who.int/GlobalAtlas/predefined. Reports/TB/PDF_Files/EG_2004_Brief.pdf.

2. Kir A, Tahaoglu K, Okur E, Hatipoglu T. Role of surgery in multi-drug resistant tuberculosis: results of 27 cases. Eur J Cardiothorac Surg. $1997 ; 12: 531-4$
3. Langston H, Barker W, Pyle M. Surgery in pulmonary tuberculosis: 11 year review of indications and results. Ann Surg. 1966;164:567-73.

4. Pomerantz BJ, Cleveland JC Jr, Olson HK, Pomerantz M. Pulmonary resection for multi-drug resistant tuberculosis. $J$ Thorac Cardiovasc Surg. 2001;121:448-53.

5. Iseman MD, Madsen L, Goble M, Pomerantz M. Surgical intervention in the treatment of pulmonary disease caused by drug resistant Mycobacterium tuberculosis. Am Rev Respir Dis. 1990;328:527-532.

6. World Health Organization. Anti-tuberculosis drug resistance in the world. Report no. The WHO/IUATLD global project on antituberculosis resistant surveillance 1999-2002. Geneva: World Health Organization; 2004.

7. Hubert B, Desenclos JC, Schwoebel V. Epidemiologie actuelle de la tuberculose. Med Ther. 1995;1:7-17.

8. Goble M, Iseman MD, Madesen LA, Waite D, Ackerson L, Horsburgh CR. Treatment of 171 patients with pulmonary tuberculosis resistant to isoniazid and rifampin. $N$ Engl J Med. 1993;328:527-32.

9. Shiraishi Y, Nakajima Y, Katsuragi N, Kurai M, Takahashi N. Resectional surgery combined with chemotherapy remains the treatment of choice for multidrug-resistant tuberculosis. J Thorac Cardiovasc Surg. 2004;128:523-528.

10. Pomerantz M, Brown J. The surgical management of tuberculosis. Semin Thorac Cardiovsc Surg. 1995;7:108-111.

11. Treasure RL, Seaworth BJ. Current role of surgery in Mycobacterium tuberculosis. Ann Thorac Surg. 1991;52:1108-12.

12. Connery CP, Knoetgen J III, Anagnostopoulos CE, Svitak MV. Median sternotomy for pneumonectomy in patients with pulmonary complications of tuberculosis. Ann Thorac Surg. 2003;75:1613-7.

13. Rizzi A, Rocco G, Robustellini M, Rossi G, Della Pona C, Massera F. Results of surgical management of tuberculosis experience in 206 patients undergoing operation. Ann Thorac Surg. 1995;59:896-900.

14. Reed CE, Parker EF, Crawford FA Jr. Surgical resection for complications of pulmonary tuberculosis. Ann Thorac Surg. 1989;48: 165-7.

15. Freixinet J. Surgical indication for treatment of pulmonary tuberculosis. World J Surg. 1997;21:475-9.

16. Sung SW, Kang CH, Kim YT, Han SK, Shim YS, Kim JH. Surgery increases the chance of cure in multi-drug resistant pulmonary tuberculosis. Eur J Cardiothorac Surg. 1999;16:187-93.

17. $\mathrm{Hu}$ Y, Coates AR, Mitchison DA. Sterilizing activities of fluoroquinolones against rifampin-tolerant populations of Mycobacterium tuberculosis. Antimicrob Agents Chemother. 2003;47:653-7.

18. Sulochana S, Rahman F, Paramasivan CN. In vitro activity of fluoroquinolones against Mycobacterium tuberculosis. J Chemother. 2005 17:169-73

19. Nahid P, Pai M, Hopewell PC. Advances in the diagnosis and treatment of tuberculosis. Proc Am Thorac Soc. 2006;3:103-10. 